Información farmacológica

RETIROS DEL MERCADO Se retira la tolcapona por reacciones adversas graves; se prohibe la venta de productos que contengan Kava-kava por su hepatotoxicidad; se retiran más productos que contienen ácido aristolóquico; se advierte contra el uso de la hierba Ephedra; se suspende la comercialización de gotas oculares de prednisolona y gentamicina; se retiran dializadores y se suspende su fabricación.

REACCIONES ADVERSAS Depresión y suicidio con la isotretinoína; hepatotoxicidad de la leflunomida; reacciones adversas al bupropión; neurotoxicidad del aciclovir y valaciclovir; reacciones hepáticas y cardiovasculares a la rosiglitazona; ataques de sueño con pramipexol y ropinirol; anemia aplásica mortal con alopurinol; probables reacciones adversas hematológicas al omeprazol y a la ranitidina.

USO RACIONAL Seguridad de los envases de paracetamol; corticoides inhalados y densidad mineral ósea; el sildenafil no debe usarse con nitratos.

ENMIENDAS A LA ROTULACIÓN

Advertencias sobre el droperidol; advertencias, contraindicaciones y precauciones sobre la nefazodona; precauciones con la levocarnitina; cambios en el prospecto de la claritromicina; cambios en el prospecto del miconazol debido a posibles interacciones con la warfarina; actualización de la información de prescripción de tioridazina por el riesgo de prolongación del intervalo QT.

EVALUACIÓN DE NUEVOS PRODUCTOS POLÍTICAS DE MEDICAMENTOS
Sibutramina; galantamina.

Armonización de la farmacovigilancia internacional.

\section{RETIROS DEL MERCADO}

\section{Se retira la tolcapona por reacciones adversas graves (Unión Europea)}

En 1997, la Comisión Europea concedió a los laboratorios Roche la autorización de comercializar la tolcapona $\left(\right.$ Tasmar $^{\circledR}$ ) para el tratamiento de la enfermedad de Parkinson, pero el Comité de Especialidades Farmacéuticas (Committee for Proprietary Medicinal Products) de la Agencia Europea de Evaluación de Medicamentos (European Medicines Evaluation Agency: EMEA) ha recomendado suspender dicha autorización por considerar que no se puede mantener su uso clínico normal en condiciones de seguridad. La decisión se basó en el hecho de que el medicamento ha producido reacciones adversas hepáticas graves, a veces mortales, que no se pueden prever mediante la realización de pruebas funcionales hepáticas. Teniendo en cuenta la hepatotoxicidad, así como la posible aparición de rabdomiólisis y síndrome neuroléptico maligno, se consideró que la relación entre los riesgos y los beneficios es desfavorable en la indicación autorizada y que no es posible restringir las indicaciones lo suficiente como para permitir un uso seguro.

Se prohibe la venta de productos que contengan Kava-kava por su hepatotoxicidad (Brasil, Canadá, Estados Unidos, Europa)

La Administración de Alimentos y Medicamientos (Food and Drug Administration: FDA) de Estados Unidos
(EE. UU.), las autoridades sanitarias canadienses (Health Canada/Santé Canadá: HC/SC) y la Agencia Nacional de Vigilancia Sanitaria de Brasil han alertado de posibles reacciones adversas graves asociadas al Kava-kava (Piper methysticum).

Los productos que contienen extractos de la hierba conocida como Kava-kava (Piper methysticum), utilizados en casos de ansiedad, estrés, insomnio y síndrome posmenstrual, se han asociado a graves reacciones hepáticas en Suiza y Alemania. En estos países se han registrado aproximadamente 25 casos de hepatotoxicidad (hepatitis, cirrosis e insuficiencia hepática), uno de los cuales necesitó trasplante hepático. Esto ha llevado a las autoridades sanitarias de ambos países a prohibir la venta de todos los productos que contengan Kava-kava. 


\section{Se retiran más productos que contienen ácido aristolóquico (Canadá y EE.UU.)}

El ácido aristolóquico, presente en diferentes plantas, es un potente cancerígeno y nefrotóxico. La FDA ha retirado del mercado 13 productos herbarios que contienen ácido aristolóquico. A su vez, HC/SC ha advertido a los consumidores de que no deben usar el producto pediátrico denominado Tao Chih Pien, que se vende en comprimidos como diurético y laxante. A pesar de que el prospecto no dice que contenga ácido aristolóquico, el prospecto chino dice que contiene $M u$ Tong, término tradicional usado para designar numerosas hierbas, entre ellas la Aristolochia. Análisis posteriores revelaron que, de hecho, Tao Chih Pien contiene ácido aristolóquico, por lo que se ha advertido a quienes estén en su posesión que no deben consumirlo; asimismo, se han tomado medidas para impedir su importación y venta.

\section{Se advierte contra el uso de la hierba Ephedra (Canadá)}

$\mathrm{HC} / \mathrm{SC}$ ha advertido a los consumidores de que no deben usar la hierba Ephedra, ni sola ni combinada con cafeína $\mathrm{u}$ otros estimulantes. Entre los productos afectados por esta advertencia se encuentran todos los que contengan en su composición ma huang, Ephedra china, extracto de ma huang, Ephedra, Ephedra sinica, extracto de Ephedra, polvo de hierba Ephedra, Sida cordifolia o epitonina. El consumo de estos productos, solos o combinados con cafeína $u$ otros estimulantes, puede causar graves reacciones adversas, a veces mortales.

\section{Se suspende la comercialización de gotas oculares de prednisolona y gentamicina (Irlanda)}

La Comisión Irlandesa del Medicamento (Irish Medicines Board) ha enviado a las autoridades sanitarias de Alemania, Botswana, Chile, Namíbia y Zimbabwe una notificación de alerta rápida de un defecto de calidad en las gotas oftálmicas de Pred-G, una suspensión que contiene prednisolona $\mathrm{y}$ gentamicina. Aunque el contenido de conservante cumplía las especificaciones al final del período de validez del producto en todos los lotes probados, algunos no cumplían los criterios de la Farmacopea Europea en la prueba de eficacia de conservantes. En espera de los resultados de nuevas investigaciones, la autorización de comercialización ha sido suspendida en Irlanda, país de fabricación, y el producto ha sido retirado del mercado en los países antes mencionados.

\section{Retiro del mercado y suspensión de fabricación de dializadores de Baxter (Unión Europea, EE.UU.)}

La FDA está investigando la muerte de más de 50 pacientes en diferentes países (Alemania, Colombia, Croacia, EE.UU., España y Taiwan), que puede haber sido causada por dializadores fabricados por Baxter Healthcare Corp. (Deerfield, IL, EE.UU.). La mayoría de los fallecidos presentaron disnea, paro cardíaco o síntomas de accidente vásculocerebral a las pocas horas de la diálisis. Investigaciones preliminares realizadas por el fabricante han llevado a concluir que en estas muertes podría estar implicado un líquido con perfluorohidrocarbono usado en el proceso de fabricación, y se están realizando pruebas confirmatorias. Los dializadores implicados, de las marcas Althane o Baxter (series A11, A15, A18, A22, AF150, AF180, AF220, AX1500 y AX2200), fueron distribuidos en más de 50 países entre enero de 1998 y octubre de 2001, fecha en la que fueron retirados del mercado por el propio fabricante, que comunicó haber suspendido su fabricación.

\section{REACCIONES ADVERSAS}

\section{Isotretinoína (España)}

La isotretinoína (Roacutan ${ }^{\circledR}$, Isotrex ${ }^{\circledR}$ ) es un retinoide que reduce la producción de sebo y se utiliza en el acné quístico grave que no responde al tra- tamiento con antibióticos convencional en hombres ni al tratamiento hormonal en mujeres. Es un medicamento sometido al régimen de "especial control médico", debido al riesgo de malformaciones congénitas graves. En los últimos años se han notificado en diversos países (Australia, EE.UU., España, Reino Unido) casos de depresión, e incluso de suicidio, atribuidos a su uso. En los EE.UU., entre 1982 y 2000, la FDA fue notificada de 431 casos de depresión, ideación suicida, intento de suicidio o suicidio (37 casos) en pacientes tratados con isotretinoína. Las edades oscilaron entre 13 y 32 años (mediana de 17) y la dosis máxima mediana fue de $1 \mathrm{mg} / \mathrm{kg} /$ día, cuando la recomendada es de 0,5 a $2 \mathrm{mg} / \mathrm{kg} /$ día.

La FDA también ha tenido conocimiento de otros 110 casos de ingreso hospitalario por depresión, ideación suicida o intento de suicidio en 85 pacientes que tomaban isotretinoína y 25 que habían dejado de tomarla hacía poco. La edad y la dosis eran similares a las de la serie antes mencionada. El cuadro mejoró en muchos casos tras la suspensión del tratamiento, pero en otros persistió. Cuatro volvieron a recibir isotretinoína tras la resolución de la depresión; los síntomas reaparecieron en uno, mientras que los otros tres pudieron seguir tomando isotretinoína, aunque a dosis más bajas, evitando el consumo de alcohol y, uno de ellos, tomando un antidepresivo. Por último, la FDA ha tenido conocimiento de otros 284 casos de depresión que no necesitaron hospitalización, 149 de ellos acompañados de otros efectos indeseados somáticos.

En principio, la aparición de síntomas de depresión en un paciente tratado con isotretinoína debe llevar a pensar que la causa es el fármaco y a suspender su administración. Si se considera que la isotretrinoína es necesaria, se puede intentar reiniciar el tratamiento a dosis más bajas.

\section{Leflunomida (Australia, España)}

La leflunomida (Arava ${ }^{\circledR}$ ) es un derivado isoxazólico que inhibe la síntesis de pirimidina por acción sobre la 
dihidroorotato-deshidrogenasa y tiene actividad antiproliferativa sobre los linfocitos T, así como actividad antiinflamatoria. En septiembre de 1999 la EMEA aprobó el uso de la leflunomida en el tratamiento de adultos con artritis reumatoidea. Hasta ahora, la EMEA ha tenido conocimiento de 295 casos de alteraciones hepáticas asociadas a su uso, sobre una exposición global de 104000 años-paciente de tratamiento. De esos 295 casos, 121 se consideraron graves y 9 fueron mortales. Todos fueron diagnosticados en los primeros 6 meses de tratamiento y cerca del $80 \%$ de los casos graves ocurrieron en pacientes que tomaban simultáneamente algún otro medicamento potencialmente hepatotóxico (principalmente metotrexato), mientras que el $27 \%$ presentaba otros factores de riesgo de hepatopatía. La leflunomida también se ha visto implicada ocasionalmente en casos de pancitopenia y reacciones cutáneas graves, como síndrome de Stevens-Johnson o necrólisis epidérmica tóxica.

En Australia, desde su comercialización a principios del año 2000, la leflunomida se ha asociado a 191 casos con sospecha de reacción adversa al fármaco. En muchos de ellos el paciente también estaba tomando metotrexato, a pesar de que la leflunomida solo está aprobada como monoterapia. El Comité Asesor de Reacciones Adversas a Fármacos (Adverse Drug Reactions Advisory Committee: ADRAC) de Australia ha señalado que los médicos deben saber que el uso simultáneo de leflunomida y metotrexato puede predisponer a la aparición de reacciones adversas al fármaco.

\section{Reacciones adversas al bupropión (Australia, Reino Unido, Singapur)}

El bupropión es un antidepresivo que a finales del año 2000 se empezó a utilizar ampliamente en pacientes dependientes de la nicotina que quieren dejar de fumar. Desde noviembre de ese año, el ADRAC de Australia ha recibido 780 notificaciones de sospecha de reacciones adversas al bupropión, en su mayoría consistentes en reaccio- nes de hipersensibilidad o efectos neurológicos o psiquiátricos. En el Reino Unido, desde finales de septiembre de 2001 se han recibido 6570 notificaciones similares, aunque se destaca que podrían estar asociadas a otros factores, como la abstinencia de la nicotina o la presencia de enfermedades o medicaciones simultáneas. La Unidad de Farmacovigilancia de Singapur también ha sido notificada de reacciones adversas al bupropión. Teniendo en cuenta estos datos, el fabricante de Zyban ${ }^{\circledR}$ y Wellbutrin SR ${ }^{\circledR}$ (GlaxoSmithKline) ha enviado a los profesionales sanitarios una carta en la que se reiteran las contraindicaciones, las interacciones farmacológicas, el riesgo de convulsiones y las precauciones que se deben tomar al administrar bupropión.

\section{Neurotoxicidad del aciclovir y valaciclovir (Suecia)}

El aciclovir es un antivírico utilizado en el tratamiento del herpes simple, de la varicela-zóster y de otras infecciones víricas. Según la Agencia Sueca del Medicamento (Swedish Medical Products Agency), hasta noviembre de 2000, 54 de las 96 reacciones adversas al aciclovir que le habían sido notificadas incluían manifestaciones neuropsiquiátricas tales como confusión, alucinaciones, psicosis, agitación, convulsiones, trastornos de la coordinación, etc. En 20 de los 27 casos de reacciones adversas al valaciclovir, el profármaco del aciclovir, se observaron manifestaciones similares. Muchos de los pacientes afectados eran mayores de 60 años y tenían insuficiencia renal.

\section{Reacciones hepáticas y cardiovasculares a la rosiglitazona (Canadá)}

Entre marzo de 2000 y febrero de 2001, el Programa Canadiense de Vigilancia de Reacciones Adversas a Fármacos (Canadian Adverse Drug Reaction Monitoring Program: CADRMP) ha sido notificado de 166 reacciones adversas a la rosiglitazona $\left(\right.$ Avandia $\left.^{\circledR}\right), 38$ de las cuales (20 cardiovasculares, 10 hepa- tobiliares y 8 hematológicas) se consideraron graves. Los trastornos hematológicos han incluido anemia, anemia ferropénica, disminución de la concentración de hemoglobina, neutropenia, leucopenia, pancitopenia, disminución de la producción de plaquetas, trombocitopenia y prolongación del tiempo de protrombina. Para reducir el riesgo de reacciones adversas cardiovasculares y hepáticas, los médicos deberían cumplir todas las recomendaciones relacionadas con el uso del fármaco y ser cautelosos al prescribir rosiglitazona a pacientes con retención de líquidos, ligera elevación de las enzimas hepáticas o enfermedades cardíacas subyacentes. Además, a los pacientes tratados con este fármaco se les debe advertir de la necesidad de estar atentos a la posible aparición de síntomas de insuficiencia cardíaca congestiva o de trastornos hepáticos.

\section{Ataques de sueño con pramipexol y ropinirol (Canadá)}

El CADRMP ha sido implicado en una alta proporción de casos de trastornos del sueño, que es uno de los efectos adversos del pramipexol (Mirapex ${ }^{\circledR}$, Boheringer Ingelheim) y del ropinirol (Requip ${ }^{\circledR}$, SmithKline Beecham): 26 de 57 y 16 de 17 casos, respectivamente. En algunos de los casos, los ataques de sueño sobrevinieron mientras el paciente conducía. Habitualmente los episodios no duran más de algunos segundos y a menudo son precedidos por síntomas premonitorios, tales como fatiga inusual. Los fabricantes de ambos fármacos ya habían advertido antes de que los pacientes no deben conducir ni realizar otras actividades que requieran estar despierto.

\section{Anemia aplásica mortal con alopurinol (Suecia)}

Hasta noviembre de 2000 se habían registrado en la base de datos sueca de reacciones adversas a fármacos 311 notificaciones relacionadas con el alopurinol en las que se detallaban 423 reaccio- 
nes adversas a este fármaco antigotoso. Las más frecuentes consistieron en reacciones cutáneas (220 casos), discrasias sanguíneas (69), síntomas y signos generales (40), trastornos hepatobiliares (35) y efectos gastrointestinales (19). Entre las discrasias sanguíneas hubo 6 casos de anemia aplásica mortal. En pacientes con lesiones renales es importante ajustar la dosis de alopurinol según la función renal. En estos pacientes se deben efectuar controles hematológicos, suspendiéndose la administración del alopurinol en caso de que aparezcan signos de discrasia sanguínea y mielosupresión.

\section{Probables reacciones adversas hematológicas al omeprazol y a la ranitidina (Suecia)}

El tratamiento prolongado con omeprazol $\left(\right.$ Losec $\left.^{\circledR}\right)$ se ha asociado a malabsorción del hierro y a la consiguiente anemia en un varón de 44 años. El paciente había estado recibiendo omeprazol durante varios años, pero no se especificaron las dosis ni la indicación terapéutica. La absorción de hierro mejoró notablemente tras la suspensión del tratamiento.

En un varón de 68 años con gastritis recurrente, el tratamiento con ranitidina (Zantac ${ }^{\circledR}$ ) se asoció a la aparición de anemia hemolítica. Se suspendió la ranitidina, se inició un tratamiento con cortisona y 4 meses más tarde la hemoglobinemia y el recuento de reticulocitos se habían normalizado.

\section{USO RACIONAL}

\section{Seguridad de los envases de paracetamol (Reino Unido)}

El principal riesgo de la intoxicación aguda por paracetamol es la hepatotoxicidad. Hay varias medidas destinadas a evitar la sobredosis intencional, entre ellas la limitación de la dosis total por envase, una vez que la mayoría de las personas que intentan suicidarse raramente toman el contenido de más de un envase. En el Reino Unido, en septiembre de 1998 se prohi- bió la venta de más de 8 g (16 dosis unitarias de $500 \mathrm{mg}$ ) a un solo paciente y de una sola vez en establecimientos distintos de las farmacias. En las farmacias se prohibió la venta de más de $16 \mathrm{~g}$ sin receta médica y de más de 50 $\mathrm{g}$ con receta médica. Varios estudios recientes han investigado la eficacia de esta medida, comparando los datos del año posterior a su adopción (septiembre de 1998 a septiembre de 1999) con las medias de los 2 años anteriores. En uno de ellos, las muertes por suicidio atribuidas al paracetamol disminuyeron en un $21 \%(P=0,01)$ en Inglaterra y Gales, los ingresos hospitalarios en un 30\%, y los trasplantes hepáticos por sobredosis de paracetamol en un $66 \%(P<0,001)$. Los resultados de este estudio y de otros similares llevaron a concluir que la reducción del riesgo de intoxicación por paracetamol se debió sobre todo a la reducción de la dosis total por envase. Se podrían adoptar medidas similares con otros fármacos vendidos en envases que contienen dosis totales potencialmente mortales.

\section{Corticoesteroides inhalados y densidad mineral ósea}

Los estudios acerca de los efectos de los corticoesteroides inhalados (CI) sobre la densidad mineral ósea (DMO) han proporcionado resultados variables y difíciles de interpretar y de extrapolar a la práctica clínica. No obstante, los datos disponibles no parecen indicar que haya un riesgo importante de efectos adversos óseos con ningún $\mathrm{CI}$ a dosis bajas o intermedias $(<1000$ $\mathrm{mg}$ /día) y, en principio, se recomienda utilizar la mínima dosis eficaz para controlar el asma. Si fuera necesaria la administración prolongada de dosis > $1000 \mathrm{mg} /$ día, parece razonable adoptar medidas preventivas ante el riesgo de osteoporosis, tales como el ejercicio físico regular, la dieta adecuada, la administración de suplementos de calcio o vitamina D, no fumar, etc. Algunos autores recomiendan determinar la DMO al principio del tratamiento y al cabo de 1 año, o realizar controles periódicos cuando la dosis acumulada supere los 5000 mg y considerar la adopción de medidas adicionales (tratamiento con estrógenos o bifosfonatos) en función de los resultados. Por último, una vez que se hayan controlado los síntomas asmáticos, se debe considerar la reducción gradual de la dosis de CI. En mujeres postmenopáusicas se recomienda asociar el tratamiento de reposición de estrógenos al tratamiento con CI. En niños parece razonable una vigilancia estrecha durante el tratamiento prolongado con CI. De cualquier modo, el riesgo de efectos adversos óseos con los CI es considerablemente inferior al de los corticoesteroides orales a dosis equivalentes, y la relación entre los riesgos y los beneficios claramente superior. Por consiguiente, se debe seguir considerando a los CI como una opción de primera línea en el tratamiento del asma crónica en niños y adultos.

\section{El sildenafilo no debe usarse con nitratos (Nueva Zelanda)}

El organismo neozelandés responsable de la seguridad de los medicamentos y de los dispositivos médicos (Medicines and Medical Devices Safety Authority: Medsafe) ha destacado la importancia de preguntar a los pacientes si están tomando sildenafilo (Viagra ${ }^{\circledR}$ ) antes de administrarles cualquier formulación que contenga nitratos y de recordarles que no deben tomar sildenafilo mientras estén recibiendo nitratos. Como el sildenafilo está contraindicado en pacientes que estén tomando nitratos por padecer enfermedades cardiovasculares, antes de prescribirlo también se les debe preguntar si toman nitratos de forma regular o intermitente, incluido el uso recreativo.

Los pacientes que tomen sildenafilo y presenten angina de pecho aguda deben ser tratados con analgésicos y otros tratamientos antianginosos. En caso de dolor torácico grave o complicado se debe consultar al cardiólogo y proceder a la hospitalización inmediata. Aunque se ha señalado que la administración de nitratos $24 \mathrm{~h}$ después del sildenafilo es segura, hay que tener en cuenta los factores que pueden 
retrasar el aclaramiento del sildenafilo (edad $>65$ años, deterioro de la función hepática o renal, tratamiento simultáneo con inhibidores de la CYP3A4) y que, por consiguiente, prolongan el tiempo durante el cual no se deben administrar nitratos. En caso de duda sobre si es seguro o no administrarle nitratos a un paciente tratado con sildenafilo, consúltese con un cardiólogo o con un especialista en urgencias.

La administración simultánea de sildenafilo y nitratos puede causar efectos vasodilatadores aditivos que produzcan hipotensión grave, potencialmente mortal. Si se decide administrar nitratos a un paciente que haya recibido sildenafilo o si se administran ambos fármacos de forma simultánea e inadvertida, el paciente debe ser vigilado cuidadosamente en servicios de emergencia que dispongan de medios de reanimación.

\section{ENMIENDAS A LA ROTULACIÓN}

\section{La FDA refuerza las advertencias sobre el droperidol (EE.UU.)}

La FDA ha reforzado las secciones de "Advertencias" y "Precauciones" del prospecto de droperidol, un tranquilizante usado sobre todo como medicación preanestésica, como tratamiento de la náusea tras la anestesia y como sedante en pacientes agitados. El droperidol se ha asociado a arritmias cardíacas mortales. Los cambios realizados incluyen una advertencia enmarcada en negro, que es el tipo de advertencia más seria que se hace en los productos aprobados por la FDA. La advertencia está destinada a llamar la atención de los médicos a la necesidad de vigilar el riesgo de arritmias cardíacas durante la administración del fármaco y a que consideren el uso de medicaciones alternativas en pacientes con alto riesgo de arritmias cardíacas. La FDA seguirá estudiando los datos de la farmacovigilancia del droperidol por si fuera necesario tomar nuevas medidas. El fabricante (Akorn Pharmaceuticals) ha enviado cartas a los médicos, farmacéuticos y otros profesionales sanitarios de los EE.UU. en las que explica las advertencias enmarcadas en negro y destaca el riesgo de prolongación del intervalo QT y de taquicardia helicoidal (torsade de pointes) cuando se administra este fármaco.

\section{La FDA refuerza las advertencias, contraindicaciones y precauciones sobre la nefazodona (EE.UU.)}

La FDA y el fabricante (BristolMyers Squibb) han añadido una advertencia enmarcada en negro y han reforzado las secciones de "Advertencias", "Contraindicaciones" y "Precauciones" del prospecto de Serzone ${ }^{\circledR}$ (nefazodona), un medicamento antidepresivo con el que se han descrito casos raros de insuficiencia hepática que ha llevado a la muerte o a la realización de trasplantes hepáticos. Con el envase se proporciona un nuevo "Prospecto para el paciente". En general no se debe iniciar el tratamiento con Serzone ${ }^{\circledR}$ en individuos con hepatopatía activa o con concentraciones séricas elevadas de transaminasas en condiciones basales. Aunque no hay pruebas de que las hepatopatías preexistentes incrementen el riesgo de sufrir insuficiencia hepática, la presencia de alteraciones en condiciones basales puede complicar la vigilancia del paciente. A los pacientes se les debe advertir de la necesidad de estar atentos a la aparición de síntomas de disfunción hepática (ictericia, anorexia, molestias gastrointestinales, malestar, etc.) y de comunicárselas inmediatamente a su médico.

\section{Se revisan las precauciones relacionadas con la administración de levocarnitina (EE.UU.)}

El fabricante de Carnitor ${ }^{\circledR}$ (levocarnitina), el laboratorio Sigma Tau Pharmaceuticals, ha enviado a los profesionales sanitarios una carta en la que destaca que el uso de este fármaco en pacientes con insuficiencia renal terminal solo está aprobado por vía intravenosa. La empresa ha recibido varias notificaciones de que se les está administrando levocarnitina por vía oral a pacientes con insuficiencia renal terminal y ha considerado que es necesario hacer los cambios pertinentes en la sección de "Precauciones" del prospecto del medicamento.

\section{Cambios en el prospecto de la claritromicina (Japón)}

El Ministerio de Salud, Trabajo y Bienestar de Japón ha pedido que se incluyan la disfunción hepática, la ictericia y la rabdomiólisis en la sección de "Reacciones adversas clínicamente importantes" de dos productos de uso pediátrico que contienen claritromicina (Clarith ${ }^{\circledR}$ y Klaricid ${ }^{\circledR}$ ). Anteriormente, varios trastornos hepáticos estaban incluidos en la sección "Otras reacciones adversas", y la rabdomiólisis en la sección "Interacciones farmacológicas" como una interacción con los inhibidores de la HMG-CoA reductasa (estatinas). Este cambio en el prospecto ha sido motivado por 23 casos de disfunción hepática y 6 de rabdomiólisis asociados al uso de claritromicina, notificados desde 1996.

\section{Cambios en el prospecto del miconazol debido a posibles interacciones con la warfarina (Canadá)}

El miconazol es un antifúngico que se puede comprar en forma de crema o supositorios vaginales sin necesidad de receta médica. La FDA y la base de datos canadiense de reacciones adversas han recibido notificaciones de que las mujeres tratadas simultáneamente con miconazol vaginal y anticoagulantes como la warfarina presentan prolongación del tiempo parcial de tromboplastina y del tiempo de protrombina. Esto ha llevado a HC/SC a solicitar a los fabricantes de miconazol vaginal que añadan una nueva advertencia a la monografía y al prospecto de sus productos. La advertencia dirá que quienes estén tomando anticoagulantes como la warfarina deberán consultar a su médico o farmacéutico antes de usar miconazol, debido al riesgo de hemorragias o equimosis. 


\section{Actualización de la información de prescripción de tioridazina por el riesgo de prolongación del intervalo QT (Nueva Zelanda)}

La información para la prescripción de tioridazina (Melleril ${ }^{\circledR}$, Aldazine $^{\circledR}$ ) ha sido actualizada en Nueva Zelanda según las recomendaciones del Comité de Reacciones Adversas a los Medicamentos (Medicines Adverse Reactions Committee). Dichas recomendaciones se basaron en los resultados de un informe de expertos encargado por Medsafe en el que se consideraba que sería prudente adoptar medidas para reducir el riesgo de prolongación del intervalo QT en pacientes tratados con tioridazina. En la actualidad, la tioridazina está contraindicada en presencia de los siguientes factores de riesgo de arritmia:

- uso de fármacos que inhiben el metabolismo de la tioridazina (por ejemplo, cimetidina, pindolol, propranolol y la mayoría de los antidepresivos);

- uso de fármacos que se sabe que se asocian a la prolongación del intervalo QT (por ejemplo, cisaprida, la mayoría de los antiarrítmicos y algunos antipsicóticos);

- existencia de prolongación del intervalo QT (QT corregido $=500 \mathrm{mseg}$ ) o de factores que predispongan al paciente a la prolongación del intervalo QT.

El tratamiento con tioridazina solo debería ser iniciado por un especialista, solo como tratamiento de tercera línea, y solo después de evaluar los factores de riesgo de arritmias y de comprobar el intervalo QTc y la concentración plasmática de potasio.

\section{EVALUACIÓN DE NUEVOS PRODUCTOS}

\section{Sibutramina (Francia)}

El tratamiento de referencia para perder peso en pacientes obesos consiste en la combinación de medidas dietéticas, ejercicio e intervenciones conductuales. En la actualidad no hay ningún tratamiento farmacológico de eficacia demostrada para reducir la morbilidad y la mortalidad asociadas al peso corporal excesivo. La sibutramina es un inhibidor de la recaptación de serotonina y de noradrenalina, relacionada estructuralmente con las anfetaminas. En Francia se ha autorizado su comercialización para el tratamiento de la obesidad y del peso corporal excesivo en pacientes con factores de riesgo asociados. El expediente clínico de la sibutramina no contiene ningún ensayo clínico que haya investigado la morbilidad o la mortalidad como variables de eficacia. En los estudios seleccionados, aleatorizados, controlados con placebo y doblemente enmascarados, un elevado número de pacientes abandonaron el seguimiento, lo cual resta valor a los resultados. No obstante, en general, los resultados son concordantes: dependiendo del estudio y de la dosis, los pacientes tratados durante 6 a 12 meses con sibutramina y medidas dietéticas perdieron, por término medio, 3 a 9,1 kg más que los pacientes tratados con dieta y placebo. No obstante, volvieron a ganar peso tras el cese del tratamiento con sibutramina y el fármaco tuvo escasos o nulos efectos beneficiosos sobre la glucemia y los lípidos. Se considera que no es razonable exponer a los pacientes obesos al riesgo de efectos adversos (especialmente el aumento de la tensión arterial) con la esperanza de perder algunos kilos, sobre todo si se tiene en cuenta que el efecto adelgazante desaparece tras el cese del tratamiento.

\section{Galantamina (Francia)}

El tratamiento sintomático de referencia para la enfermedad de Alzheimer leve a moderada son los inhibidores de la colinesterasa, como el donepezilo, pero su eficacia es moderada y solo se benefician de su administración aproximadamente el $10 \%$ de los pacientes tratados. La galantamina es el cuarto inhibidor de la colinesterasa que se comercializa en Francia para el tratamiento de la enfermedad de Alzheimer. El expediente clínico contiene datos de cinco estudios doblemente enmascarados, controlados con placebo, de 3 a 6 meses de duración, pero no hay datos comparativos entre la galantamina y otros fármacos. Estos estudios han revelado que cerca de un 5 a $13 \%$ de los pacientes tratados con galantamina mejoran. Estos datos son muy similares a los obtenidos con sus predecesores, como el donepezilo o la rivastigmina, proporcionando la misma eficacia moderada frente a los síntomas y ningún impacto tangible sobre la progresión de la enfermedad. Los acontecimientos adversos son un factor limitante de las dosis. Con la dosis máxima, de 24 a $32 \mathrm{mg} /$ día, la galantamina solo tiene un efecto clínicamente manifiesto en el 5 a $13 \%$ de los pacientes. Los efectos adversos son muy frecuentes y similares a los de otros inhibidores de la colinesterasa (násuseas, vómitos, diarrea, dolor abdominal, dispepsia, etc.). Se concluye que, cuando los familiares de los pacientes con enfermedad de Alzheimer soliciten un tratamiento farmacológico, el donepezilo sigue siendo el tratamiento de referencia, a pesar de sus efectos adversos, dado que no existe ninguna opción mejor.

\section{POLÍTICAS DE MEDICAMENTOS}

\section{Armonización de la farmacovigilancia internacional}

Los fundamentos de la vigilancia internacional de las reacciones adversas a los medicamentos fueron establecidos por la Organización Mundial de la Salud (OMS) en 1968. Desde entonces, el Programa Internacional de Vigilancia Farmacéutica de la OMS ha realizado importantes progresos que han sido posibles gracias al trabajo realizado por los Centros Nacionales participantes en el programa y la colaboración con diversos organismos nacionales e internacionales, grupos de profesionales, instituciones académicas y la industria farmacéutica. La pertenencia al Programa Internacional de Vigilancia Farmacéutica de la OMS está abierta a los países que hayan es- 
tablecido un centro nacional de notificación. Cada Centro Nacional (en la actualidad hay más de 60) se compromete a cumplir los requisitos establecidos por el programa, entre ellos el envío a la base de datos de la OMS de sus datos sobre reacciones adversas a los medicamentos en el formato establecido. Esta base de datos contiene en la actualidad 2,7 millones de notificaciones y es usada sin restricciones por los Centros Nacionales, algunos de los cuales han decidido poner sus datos a disposición de organismos externos. La base de datos dispone de un servicio de búsquedas múltiples y se pueden solicitar búsquedas ad hoc realizadas por el personal del Centro Colaborador de la OMS para la Vigilancia Farmacéutica Internacional, con base en Uppsala, Suecia.

Se han armonizado criterios internacionales, como las definiciones o los procedimientos para asegurar la confidencialidad de los datos. Las definiciones son acordadas por todos los Centros Nacionales y los cambios o adiciones son hechos por consenso. También se han elaborado declaraciones de advertencia en las que se definen claramente los límites de uso e interpretación de los datos y que son vinculantes para todos los organismos externos a la OMS.

A través del Centro Colaborador y de los Centros Nacionales, la OMS es el único organismo en todo el mundo que tiene una perspectiva internacional e independiente de la seguridad de los fármacos. Sin consenso es difícil conseguir una armonización internacional que permita variaciones cuando estén justificadas, pero que asegure la estandarización, sin variaciones, mediante la aplicación de prácticas óptimas y consensuadas. Esto se está consiguiendo gracias a la OMS, cuya autorizada labor en este campo es ampliamente reconocida y aceptada.

\section{REFERENCIAS}

WHO Information Exchange System. Alert No. 104. October 2001.

FDA Talk Paper, December 2001. Disponible en http://www.fda.gov/bbs/topics

Fundació Institut Català de Farmacologia. Butlletí Groc. 2001;14:10-11.

Boletín Terapéutico Andaluz. 2001;17:13-15.

Prescrire International. 2001;10:140-141.

Prescrire International. 2001;10:180-181, 189.
WHO Pharmaceuticals Newsletter 2001;2: 1-12.

WHO Drug Information 2001;15:76-83.

EMEA-CPMP/2986-01. Disponible en http:// www.emea.eu.int

ANVISA Alerta Ufarm/Anvisa No. 14, 20/12/01. Disponible en http://www.anvisa.gov.br/ farmacovigilancia

FDA/MEDWATCH, 2002. Safety Information Summaries. Disponible en http://www. fda.gov/medwatch/SAFETY/2002

Información farmacológica da a conocer las decisiones oficiales sobre regulación de productos farmacéuticos adoptadas por organismos gubernamentales e internacionales en todo el mundo, los fundamentos científicos en que se sustentan tales decisiones y otros datos de interés relacionados con el tema. Como la mayor parte de la información proviene de fuentes de circulación relativamente limitada, su diseminación en esta forma permite hacerla llegar a un público más amplio. De esta manera se pretende contribuir a limitar el uso irracional de medicamentos y fomentar su uso racional en la Región de las Américas. La sección está a cargo del Programa de Medicamentos Esenciales y Tecnología para la Salud (HSE) de la OPS y se publica en la Revista Panamericana de Salud Públical Pan American Journal of Public Health con regularidad. Las separatas pueden solicitarse al programa mencionado, Organización Panamericana de la Salud, 525 Twenty-third Street, NW, Washington, DC 20037, EUA. 